\title{
Pyridoxine in the Treatment of Tetanus Neonatorum
}

\author{
by \\ MUSA KAZIM CAGLAR \\ (Dr. Sami Ulus Children's Hospital and Hacettepe Children's Hospital, \\ Ankara, Turkey)
}

\begin{abstract}
A prospective study has been conducted to assess the efficacy of oral pyridoxine in the treatment of neonatal tetanus. A little reduction in mortality was observed by adding pyridoxine to the conventional therapy. The mortality in this group was $50.0 \%$ as compared to $62.5 \%$ in controls. This result did not confirm the ones in the literature. This could be sourced from the fact that additional infection was $50.0 \%$.

\section{Introduction}

Tetanus neonatorum still continues to be a serious problem in all developing countries as in Turkey (Yalaz and Hasanoglu, 1978). In the many reports of conventional therapy for tetanus neonatorum in developing countries, mortality has been estimated to be over $60.0 \%$ (Salimpour, 1977; Yalaz and Hasanoglu, 1978). Mortality and morbidity of tetanus neonatorum are quite low in developed countries (Edwards and James, 1979; Golbraith et al., 1981) since modern methods like mechanical ventilators, neuromuscular blocking agents, aseptic delivery techniques and antenatal tetanus immunisation of mothers

are available for them. As the disease is self-limiting, the main aim for treatment should be to prevent its complications. Therefore, cheap and practical treatment of tetanus neonatorum becomes more important for developing countries. In the recent reports (Daud et al., 1981; Godel, 1982; Hajailay et al., 1983), trials with pyridoxine therapy for tetanus neonatorum are very encouraging. Thus, in order to assess the efficacy of oral pyridoxine therapy plus conventional therapy, a prospective study was carried out in Dr. Sami Ulus Childrens Hospital and Hacettepe Children's Hospital.
\end{abstract}




\section{Materials and Methods}

Both hospitals are referral centre for whole Turkey, but mostly for middle Anatolia. During the period of three years, from 1 August 1982 to 1 August 1985, 32 cases of neonatal tetanus were taken into the study. The diagnosis in all cases was clinical. In order to assess the severity of the disease, the system of scoring previously reported was used (Daud et al., 1981).

All patients were isolated in a separate dark and silent room. The basic treatment was conventional. Initially diazepam in a dose of $0.5 \mathrm{mg} / \mathrm{kg}$ was administered intravenously for rapid control of spasms, then a nasogastric tube was inserted to all patients for oral medications. The nutrition was maintained by intravenous fluids in the initial stages, then by milk after the intense spasms ceased. Anti-tetanus serum was

\section{Results}

Ten of control patients $(62.5 \%)$ expired at the end of the study. It was $50.0 \%$ in the pyridoxine group. The cases of pyridoxine group were matched statistically to control patients in respect of mean age of developing signs and symptoms (4.6 day in expired control patients, 4.87 days in expired pyridoxine group; and 6.16 days in survived control patients, 6.4 days in survived pyridoxine group), sex, weight, mode given in a dose of 10.000 units intramuscularly in the emergency room. Penicillin $\mathrm{G}$ in a dose of 100.000 units/ $\mathrm{kg}$ per day intravenously was the sole antibiotic agent given initially. If the patient had an additional infection as septicemia, pneumonia, omphalitis, gentamicin was added in a dose of $5 \mathrm{mg} / \mathrm{kg}$ per day intravenously to the antibiotic regimen. Diazepam was continued orally and was arranged according to the severity and frequency of the spasms. Pyridoxine was added to the treatment in 16 babies. The other half was treated with only conventional therapy. A patient was considered to be cured when two criterias were full-filled i.e. (1). absence of spasms for 48 hours after stopping sedation, (2) toleration of normal oral feeding.

of delivery (all delivered at home), the scoring system $(7,6,3$ patients of the control group and $6,7,3$ patients of the pyridoxine group were found to be in the Grade IV, III and II, respectively; there was no case in the Grade I which was mildest form.), additional infection (in both groups being $50.0 \%$ ) and duration of treatment before death (1.65 day in control group, 1.75 day in pyridoxine group). is why pyridoxine has been used in the treatment of tetanus neonatorum.

In the recent reports, Daud et al. (1981), Hajailay et al. (1983) and Godel (1982) have found a significant decrease in the mortality of tetanus neonatorum with the treatment of pyridoxine. In the study of Daud et al. (1981) mortality has decreased from $55.13 \%$ to $23.7 \%$. Although Godel (1982) has not used control group, percentage of mortality was quite low as being $15 \%$. Survival rate has increased from $23.7 \%$ to $47.4 \%$ in the study of Hajailay et al. (1983). However, this present study showed that mortality has only decreased from $62.5 \%$ to $50.0 \%$. In the other studies, pyridoxine was administered orally (Hajailay et al., 1983), intramuscularly (Godel, 1982) and intravenous continously (Daud et al., 1981). The route of administration should not be a factor for this contradiction since pyridoxine was also given by oral route in one of these studies (Hajailay et al., 1983). However, we dot not have suffi- cient information about absorption of pyridoxine during the neonatal period although it is water-soluble and readily absorbed from the gastrointestinal system. Majority of the patients in the present study were either in Grade III and IV $(81,25 \%)$ which was comparable to other studies in which percentage of severe forms of disease was $88.2 \%$ (Hajailay et al., 1983) and $100 \%$ (Daud et al., 1981). It was evident in the study of Hajailay et al. that convulsions could be controlled earlier in pyridoxine group as compared to that of control group. However, this was not obvious in the present study. In all previously reported studies, it has been found that survival rate was higher in all grades of pyridoxine group when compared to controls, but this finding was not confirmed in that present study. This could be sourced from the fact that additional infection rate was $50.0 \%$ in the present study, which was not noted in the previous reports.

\section{Conclusion}

Present study shows that there is not it is low, might be assumed to be worthy significantly difference between control and pyridoxine groups from the point of rognostic factors. Therefore, reduction in mortality from $62.5 \%$ to $50.0 \%$, although in the treatment of tetanus neonatorum, since it could be sourced from the fact that additional infection was quite high, being $50,0 \%$ in the present study.

\section{Discussion}

It has been known that tetanus toxin of GABA and glycine at the nerve ending causes spasms. Tetanus toxin supresses the should increase inhibitory responses and release of the inhibitory neurotransmitters, reduce spasms. Pyridoxine is known to be Gamma-Amino-n-Butyric Acid (GABA) a co-enzyme of glutamate decarboxylase and glycine (Benecke et al., 1977). Since which is necessary in the production of both GABA and glycine are normally ef- GABA from glutamic acid and trial of pyfective at the level of postsynaptic recep- ridoxine in rats has been shown to increase tors, any measure that increases production production of GABA (Steiner, 1969). That 


\section{REFERENCES}

1. BENECKE, R.; TAKANO, K.; SCHMIDT, J.: Tetanus toxin induced actions on spinal Renshaw cells and Ia-inhibitory interneurons during development of local tetanus in the cat. Exp. Brain Res. 27: 271 (1977).

2. DAUD, S.; MOHAMMAD, T.; AHMAD, A.: Tetanus neonatorum. J. trop. Pediat. 27: 308 (1981).

3. EDWARDS, R.A.; JAMES, B.: Tetanus and psychiatry: Unexpected bed fellows. Med. J. Aust. 1: 483 (1979).

4. GODEL, J.C.: Trial of pyridoxine therapy for tetanus neonatorum. J. infect. Dis. 145: 547 (1982).

5. GOLBRAITH, N.S.; FORBES, P.; TILLET, H.: National surveillance of tetanus in England and Wales. J. infect. Dis. 3: 181 (1981).

6. HAJAILAY, R.; SHARA, N.R.; AGARWAL, V.K.: Pyridoxine therapy in tetanus neonatorum. Indian. Pediatr. 20: 935 (1983).

7. SALIMPOUR, R.: Cause of death in tetanus neonatorum: Study of 233 cases with 54 necropsies. Arch. Dis. Child. 52: 587 (1977).

8. STEINER, F.A.: L-glutamic acid, gamma-aminobutyric acid and pyridoxal-5'-Phosphate at the level of the single unit in rat brain. Ann. N.Y. Acad. Sci. 166: 199 (1969).

9. YALAZ, K.; HASANOGLU, E.: Prognosis of tetanus neonatorum according to various methods of treatment. Proceedings XV International Congress of Pediatrics, 1978. 